\title{
OPEN Polynomial fitting method of background correction for electron backscatter diffraction patterns
}

\author{
Yi-Yun Tsai, Yi-Chen Pan \& Jui-Chao Kuo $\bowtie$ \\ A raw electron backscatter diffraction (EBSD) signal can be empirically decomposed into a Kikuchi \\ diffraction pattern and a smooth background. For pattern indexing, the latter is generally undesirable \\ but can reveal topographical, compositional, or diffraction contrast. In this study, we proposed a \\ new background correction method using polynomial fitting (PF) algorithm to obtain clear Kikuchi \\ diffraction patterns for some applications in nonconductive materials due to coating problems, at \\ low accelerated voltage and at rough sample surfaces and for the requirement of high pattern quality \\ in HR-EBSD. To evaluate the quality metrics of the Kikuchi patterns, we initially used three indices, \\ namely, pattern quality, Tenengrad variance, and spatial-spectral entropy-based quality to detect \\ the clarity, contrast, and noise of Kikuchi patterns obtained at 5 and $15 \mathrm{kV}$. Then, we examined the \\ performance of PF method by comparing it with pattern averaging and Fourier transform-based \\ methods. Finally, this PF background correction is demonstrated to extract the background images \\ from the blurred diffraction patterns of EBSD measurements at low $\mathrm{kV}$ accelerating voltage and with \\ coating layer, and to provide clear Kikuchi patterns successfully.
}

Recently, electron backscatter diffraction (EBSD) in scanning electron microscopy (SEM) has become a standard analysis technique in materials science because of increasing requirements of its application in ultrafine-grained nanocrystalline materials ${ }^{1-3}$. However, the application of the EBSD technique is limited by its lateral and longitudinal resolution, which can be improved by reducing the accelerating voltage, applying an energy filter ${ }^{4,5}$, and decreasing the thickness of specimens using $\mathrm{t}-\mathrm{EBSD}^{6,7}$. Steinmetz and Zaefferer measured the physical lateral resolution of $30 \mathrm{~nm}$ and $10 \mathrm{~nm}$ for twinning-induced plasticity steel samples at accelerating voltages of 15 and $7.5 \mathrm{kV}$, respectively ${ }^{8}$. Chen et al. ${ }^{9}$ reported that the best physical lateral resolution of $34.5 \mathrm{~nm}$ is obtained at an accelerating voltage of $10 \mathrm{kV}$ for copper. Tripathi and Zaefferer ${ }^{10}$ obtained the best lateral resolution of $240 \mathrm{~nm}$ at an accelerating voltage of $5 \mathrm{kV}$ for magnesium. Moreover, the reduction of the accelerating voltage is accompanied by disadvantages, such as reduced source brightness, increased chromatic aberration, increased sensitivity to stray fields, and deflection of the beam from the secondary electron collection field ${ }^{9}$. Furthermore, a decrease in the accelerating voltage decreases the total number of backscattered electrons and the electrons detected by the CCD detector 9 . Fortunately, EBSD measurements at low accelerating voltages have extraordinary advantages of limiting the charge in semi- or nonconducting samples ${ }^{10,11}$, reducing the electron-beam interaction volume in high spatial resolution mapping ${ }^{12,13}$, and capturing enhanced band contrast, higher-order bands, or inelastic scattering effects ${ }^{14,15}$.

Kikuchi patterns are formed by near-surface electrons having energy loss within $10 \%$ of the incident beam energy, called low-loss electrons ${ }^{6,16}$. The formation of backscatter Kikuchi patterns can be explained by the formation of incoherent point sources within a material and then by the subsequent elastic scattering. During the consecutive scattering events, occur single or multiple inelastic and elastic scattering occurs, leading to incoherent point sources within a material ${ }^{10,17,18}$. Consequently, electrons from these point sources experience elastic scattering at given Bragg angles to form Kikuchi bands, whereas the other electrons do not undergo elastic scattering at given Bragg angles to contribute to diffuse background ${ }^{3,4,17,19}$. Winkelmann et al. ${ }^{20}$ and Deal et al. ${ }^{21}$ stated that the influence factors on the background intensity distribution in EBSD patterns include the accelerating voltage of the incident beam and the pattern center of EBSD patterns, as well as the working distance and the tilt angle. 
EBSD patterns can be empirically divided into two partitions of Kikuchi diffraction (KD) and background $(B G)^{22-25}$. Then, the background correction method is generally used for the post-processing of EBSD patterns to solve the problem of low signal-to-noise ratio in EBSD patterns. Here, the background division and subtraction for the background correction method are used for multiplicative and additive processing of images, respectively. However, the static background division is usually applied for the image post-processing of Kikuchi patterns, that is, to generate a background through averaging Kikuchi patterns obtained from many differently oriented grains ${ }^{26}$. In addition, Winkelman ${ }^{15}$ proposed a dynamic correction method, which generates a background for each individual pattern, and Britton et al. ${ }^{27}$ reported a background construction method using a Gaussian model approach.

In the present study, we propose a new polynomial fitting $(\mathrm{PF})$ background correction method for correcting the background in EBSD patterns and providing high-quality Kikuchi patterns. This method combines the concepts of the dynamic background correction and the static background correction. The potential applications can be found in nonconductive materials, at low accelerated voltage and at rough sample surfaces, and for the requirement of high pattern quality in HR-EBSD reported by Wilkinson ${ }^{28}$. Furthermore, we compare this method with two background correction methods using pattern averaging (PA) and Fourier transform-based (FT) approaches. In addition, to evaluate the quality metrics of the Kikuchi patterns prior to indexing, we use three techniques, namely, pattern quality (PQ), Tenengrad variance (TenV), and spatial-spectral entropy-based quality (SSEQ) to detect the clarity, contrast, and noise of processed images, respectively.

\section{Results and discussion}

Evaluation of quality metrics of Kikuchi patterns. In the nonconductive material applications, at low accelerated voltage and at rough sample surfaces, EBSD measurements show blurred patterns. The quality of Kikuchi patterns has a significant influence on the performance of the indexing processing. Thus, the quality metrics should be applied to evaluate the image quality. Three techniques, namely, PQ, TenV, and SSEQ are applied to quantify the quality of images with respect to contrast, blur, and noise. First, the definition of PQ is presented as follows ${ }^{29,30}$ :

$$
P Q=\frac{1}{N} \sum_{i=1}^{N} H\left(\rho_{i}, \theta_{i}\right),
$$

where $N$ is the number of diffraction bands being five, and $H$ is the height of the Hough peak corresponding to a band at location $\left(\rho_{i}, \theta_{i}\right)$. Here, PQ is used to detect the sharpness of the observed bands, and TenV and SSEQ are used to the image quality.

Second, TenV ${ }^{31}$, which represents the gradient magnitude between pixels in an image, is given by the following:

$$
\operatorname{Ten} V(I)=\sum_{m=1}^{M} \sum_{n=1}^{N}[S(m, n)-\bar{S}]^{2} \bar{S}=\frac{1}{N M} \sum_{m=1}^{M} \sum_{n=1}^{N} S(m, n),
$$

where $S(m, n)$ represents a gradient magnitude of $I(m, n)$ pixels, and $\bar{S}$ is the mean of the magnitudes given by the following:

$$
\bar{S}=\frac{1}{N M} \sum_{m=1}^{M} \sum_{n=1}^{N} S(m, n) .
$$

The larger deviation of the gray level indicates the higher the TenV value.

Third, SSEQ is a type of no-reference image quality assessment (called NR-IQA) approach to evaluate the degree of distortion in a single image; it is based on the calculation of spectral and spatial entropy ${ }^{32}$. The SSEQ value from 0 to 100 indicates good to poor quality. The procedure of SSEQ approach can be roughly divided into four steps, as follows: down sampling process, partition image to $M \times M$ blocks, feature pooling, and prediction of the image quality score. The spatial entropy is given by the following:

$$
E_{s}=-\sum_{x} p(x) \log _{2} p(x)
$$

where $x$ is the pixel value within a block, with empirical probability density $p(x)$, i.e., relative frequency here. The spectral entropy is given by the following:

$$
E_{f}=-\sum_{i} \sum_{j} P(i, j) \log _{2} P(i, j)
$$

where $P(i, j)$ is a spectral probability map.

After introduction to three image quality metrics of PQ, TenV, and SSEQ, modifications of contrast, sharpness, and noise were carried out on a raw image in Fig. 1j. For contrast modification, the low limit of the gray level is selected at 21,42 , and 63 to fix the gray level at 255. Figure $1 \mathrm{a}-\mathrm{c}$ show that the low limit from 63 to 21 increases the image contrast because of the increasing range of the gray level ${ }^{33}$. When considering the increasing percentage with respect to the raw image, that of TenV is significantly larger than that of $P Q$, whereas SSEQ shows slightly decreasing percentage. Thus, TenV and PQ metrics are sensitive to the change in image contrast in Table 1. 


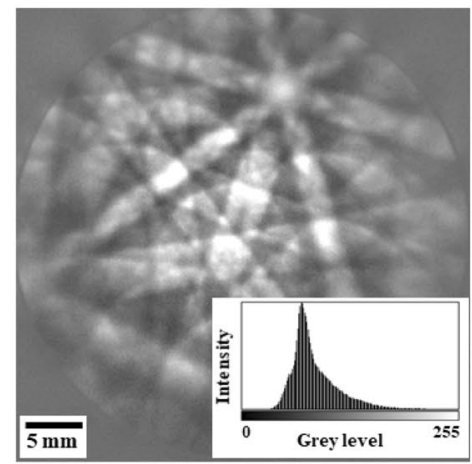

(a)

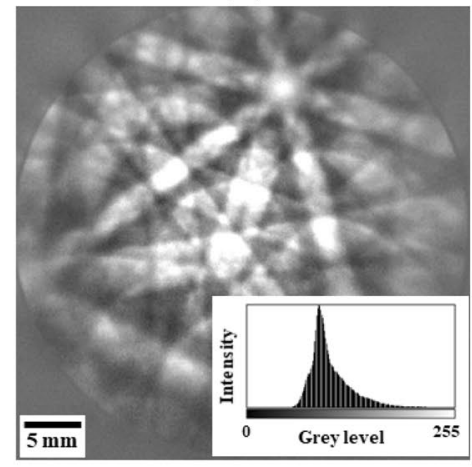

(b)

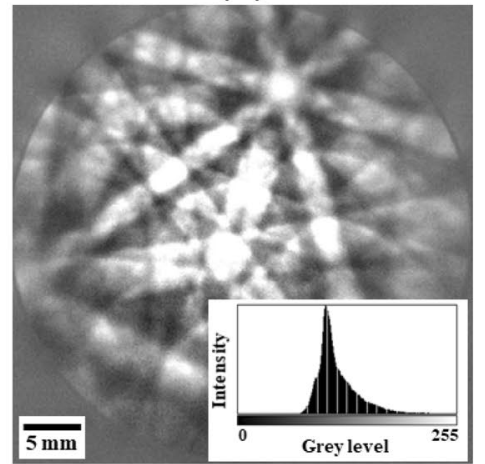

(c)

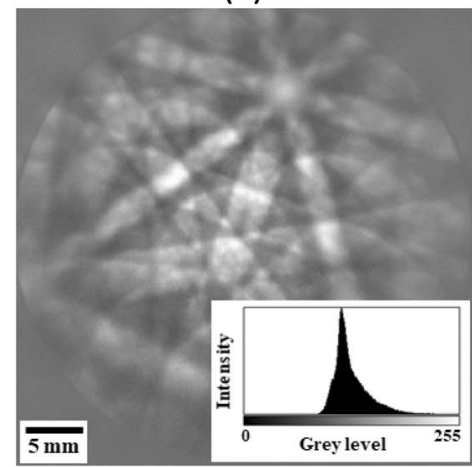

(j)

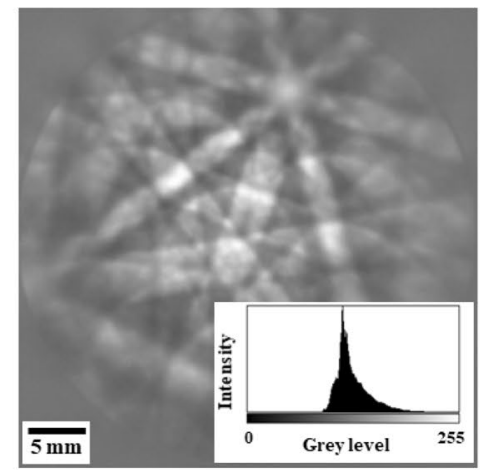

(d)

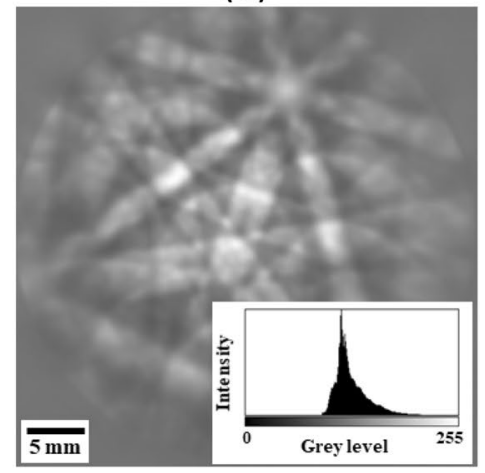

(e)

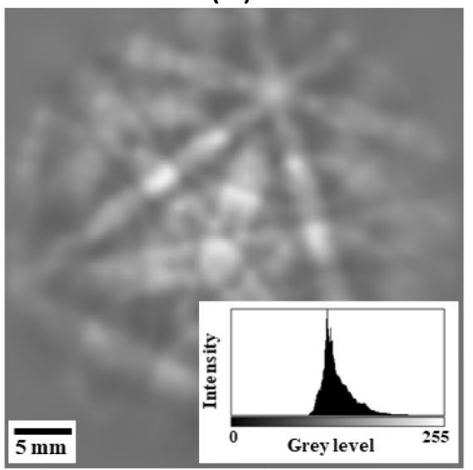

(f)

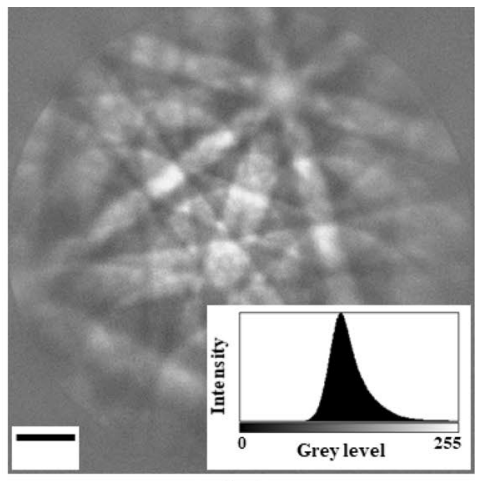

(g)

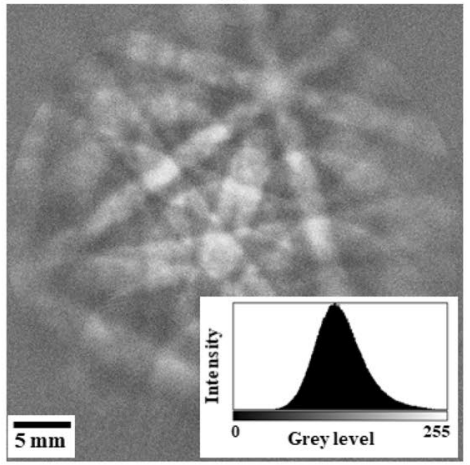

(h)

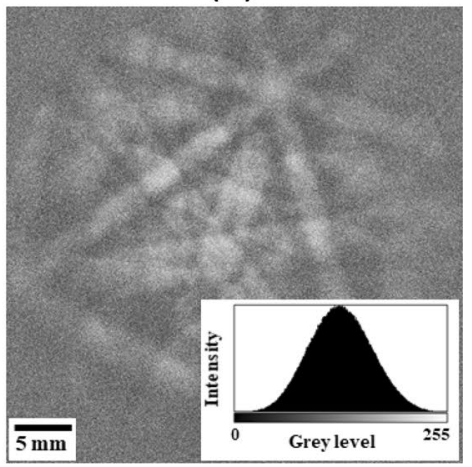

(i)

Figure 1. Contrast-modified images with the low gray level of (a) 63, (b) 42 and (c) 21; sharpness-modified images using Gaussian blur filter with the standard deviation $\sigma_{1}$ of (d) 3, (e) 5 and (f) 10; and noise-modified images using Gaussian noise with the standard deviation $\sigma_{2}$ of $(\mathbf{g}) 15$, (h) 35 and (i) 70 with respect to (j) raw image as the reference image. 


\begin{tabular}{|l|l|l|l|l|}
\hline \multicolumn{5}{|l|}{ Contrast adjustment } \\
\hline Gray level & $-^{\star}$ & $63-255$ & $42-255$ & $21-255$ \\
\hline PQ & 10,024 & 19,264 & 24,519 & 26,075 \\
\hline TenV & $7.02 \times 10^{5}$ & $2.42 \times 10^{6}$ & $6.55 \times 10^{6}$ & $1.48 \times 10^{7}$ \\
\hline SSEQ & 13.03 & 9.89 & 10.69 & 11.82 \\
\hline Blur adjustment \\
\hline$\sigma_{1}$ & $-^{\star}$ & 3 & 5 & 10 \\
\hline PQ & 10,024 & 9923 & 7728 & 7190 \\
\hline TenV & $7.02 \times 10^{5}$ & $4.02 \times 10^{4}$ & $2.39 \times 10^{4}$ & $6.43 \times 10^{3}$ \\
\hline SSEQ & 13.03 & 32.11 & 64.01 & 67.51 \\
\hline Noise adjustment & $-^{*}$ & 15 & 35 & 70 \\
\hline$\sigma_{2}$ & 10,024 & 9924 & 7729 & 7191 \\
\hline PQ & $7.02 \times 10^{5}$ & $3.46 \times 10^{7}$ & $8.58 \times 10^{8}$ & $6.01 \times 10^{9}$ \\
\hline TenV & 13.03 & 41.01 & 52.94 & 61.09 \\
\hline SSEQ & \multicolumn{5}{|l}{} \\
\hline
\end{tabular}

Table 1. Indexing values of PQ, TenV, and SSEQ after modification of contrast, blur and noise. ${ }^{\star}$ Remark: without any adjustment.

In the case of sharpness modification, a $2 \mathrm{D}$ Gaussian blur filter $\left(\mathrm{G}_{2 \mathrm{D}}\right)$ called a nonuniform low-pass filter that preserves the low spatial frequency and smooth images is expressed as follows ${ }^{34}$ :

$$
G_{2 D}=\left(x, y, \sigma_{1}\right)=\frac{1}{2 \pi \sigma^{2}} e^{-\frac{x^{2}+y^{2}}{2 \sigma_{1}^{2}}},
$$

where $x$ and $y$ are the location positions. The standard deviation $\sigma_{1}$ of the Gaussian blur filter changes the amount of blurring. When increasing the standard deviation $\sigma_{1}$ from 3 to 10, the images become more blurred, as shown in Fig. 1d-f. The increase in the standard deviation from 3 to 10 decreases the PQ value from 9923 to 7190 , but increases TenV value from $4.02 \times 10^{4}$ to $6.43 \times 10^{3}$ and the SSEQ value from 32.11 to 67.51 , as shown in Table 1 . When considering the increasing percentage with respect to the raw image, the indexing of TenV is observed to be more sensitive than that of SSEQ due to the sharpness change, but that of PQ is insensitive.

When increasing noise in images, the probability density function called Gaussian noise ${ }^{35}$ of a random Gaussian variable $z$ is given by the following:

$$
P G(z)=\frac{1}{\sigma_{2} \sqrt{2 \pi}} e^{-\frac{(z-\mu)^{2}}{2 \sigma_{2}^{2}}},
$$

where $z$ represents the gray level, $\mu$ is the mean gray value, and $\sigma_{2}$ is the standard deviation. When increasing standard deviation $\sigma_{2}$ from 15 to 70, the PG value becomes larger, indicating the broader distribution of noise in Fig. 1g-i. The increase in the standard deviation from 15 to 70 decreases the PQ value from 9924 to 7191 and TenV value from $3.46 \times 10^{7}$ to $6.01 \times 10^{9}$, but increases the SSEQ value from 41.01 to 61.09 , as shown in Table 1 . Although the indexing of Ten $\mathrm{V}$ shows a more increasing percentage here, the increasing TenV value indicates a sharp image, which is inappropriate. Here, the indexing of SSEQ is more sensitive than that of PQ to detect noise. Therefore, the TenV indexing is sensitive to change in contrast and blur and the SSEQ indexing to noise detection.

Estimation of background image by PA, FT, and PF methods. At low accelerated voltage, Kikuchi patterns reveal blurred edges with a low signal-to-noise ratio. Thus, adaptive histogram equalization (AHE) is initially used to improve contrast in images. After contrast enhancement with AHE, the comparison of the raw images in Fig. 2a,b shows an improved contrast, and the enhanced contrast broadens the range of gray level in Fig. 2c. The profile of gray level marked by the yellow line is plotted in Fig. 2a,b, and the profile is significantly increased after implantation of AHE.

Diffraction patterns contain a significant amount of background, which can be approximated by averaging patterns from various crystallites in a given polycrystalline sample or simulated patterns at low magnifications ${ }^{5}$. This process describes the first background generation method called PA, where an average pattern is revealed without crystallographic details that remove long-range intensity gradients.

In the second method called $\mathrm{FT}^{36}$, a low-pass filtering operation (also called a "blurring" or "smoothing" filter) is used to selectively smooth the image background without changing the bright areas. The 2D discrete Fourier transform of an $M \times N$ image is expressed by the following:

$$
F(u, v)=\frac{1}{M N} \sum_{x=0}^{M-1} \sum_{x=0}^{N-1} f(x, y) e^{-j 2 \pi\left(\frac{u x}{M}+\frac{v y}{N}\right)}
$$

where $x$ and $y$ are the spatial variables, and $u$ and $v$ are the frequency variables. The center region of the frequency domain represents the low frequency region, which corresponds to the background signal of Kikuchi patterns. 


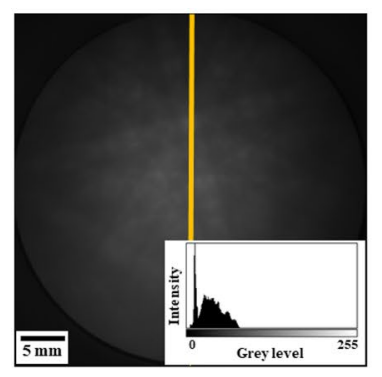

(a)

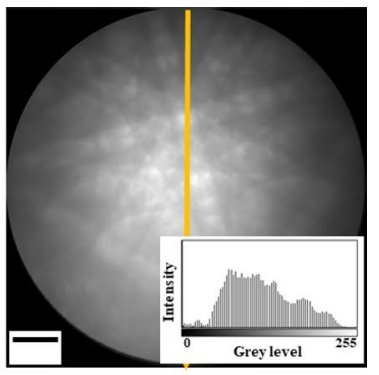

(b)

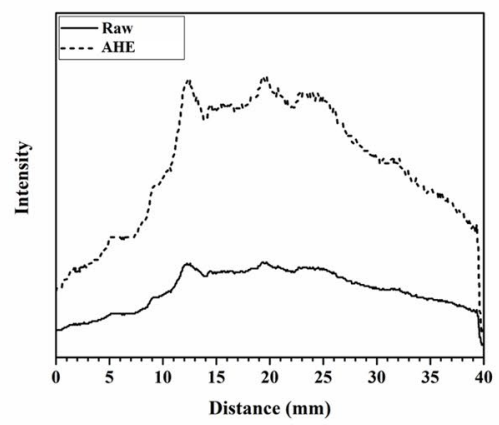

(c)
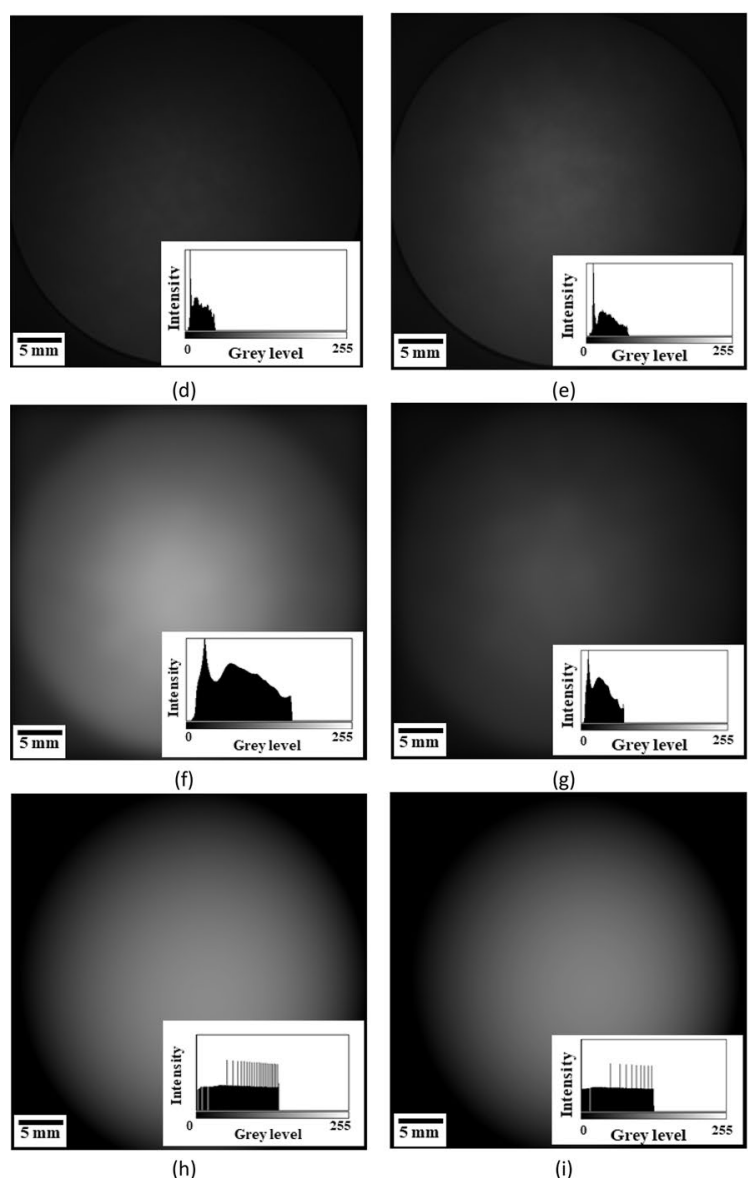

Figure 2. (a) Raw image, (b) contrast-enhanced image using AHE filter at $5 \mathrm{kV}$, and (c) the gray-level profile comparison between raw and contrast-enhanced images. Background generation by (d,e) PA, (f,g) FT, and (h,i) PF methods, where $(\mathbf{d}, \mathbf{f}, \mathbf{h})$ are for $15 \mathrm{kV}$ and $(\mathbf{e}, \mathbf{g}, \mathbf{i})$ are $5 \mathrm{kV}$. 


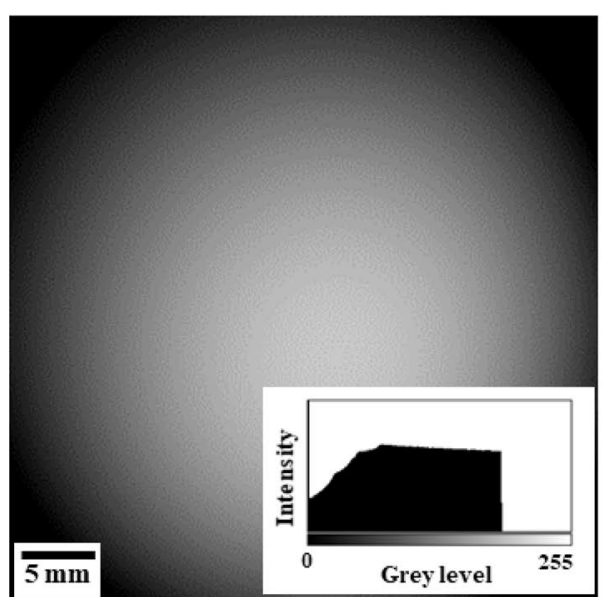

(a)

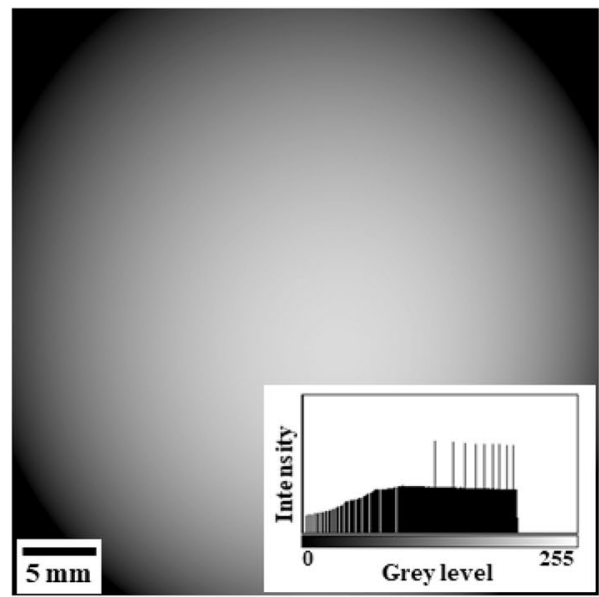

(c)

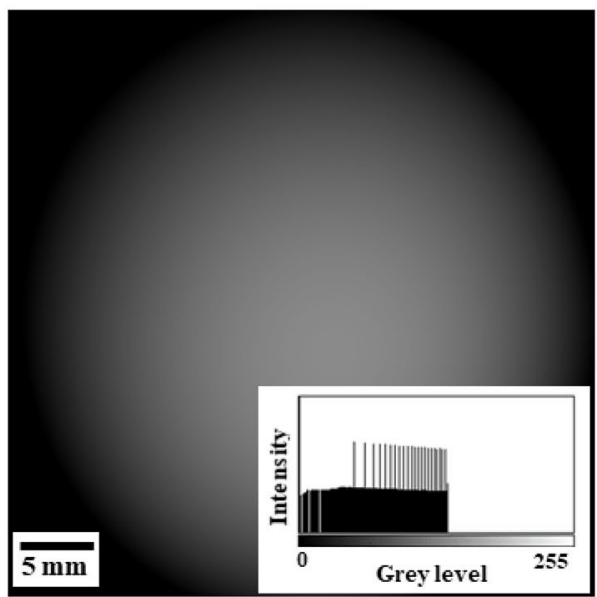

(e)

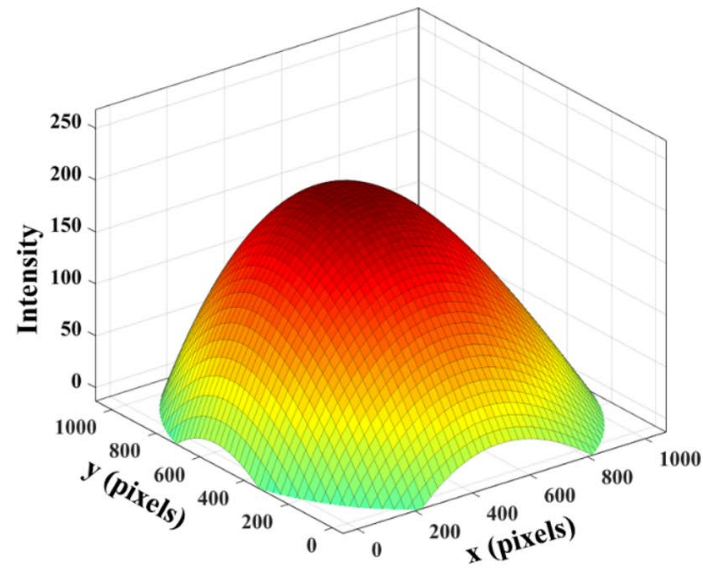

(b)

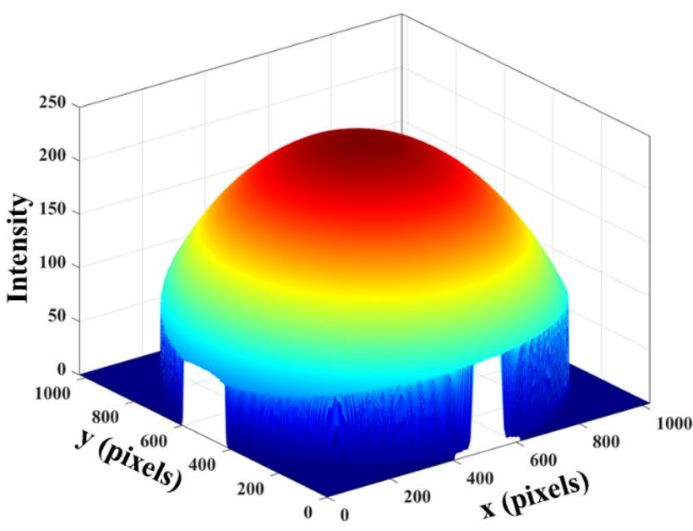

(d)

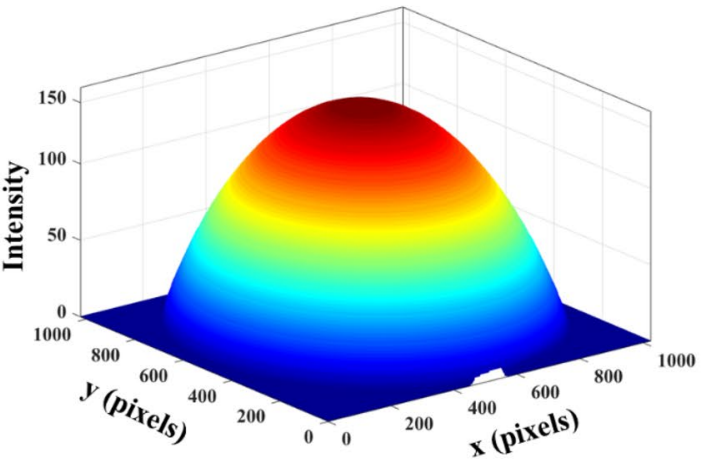

(f)

Figure 3. The images (a) without image processing, (c) after surface fitting and $\gamma$ correction, and (e) after background optimization at $15 \mathrm{kV}$, where $(\mathbf{b}, \mathbf{d}, \mathbf{f})$ are the $3 \mathrm{D}$ plots corresponding to $(\mathbf{a}, \mathbf{c}, \mathbf{e})$. 


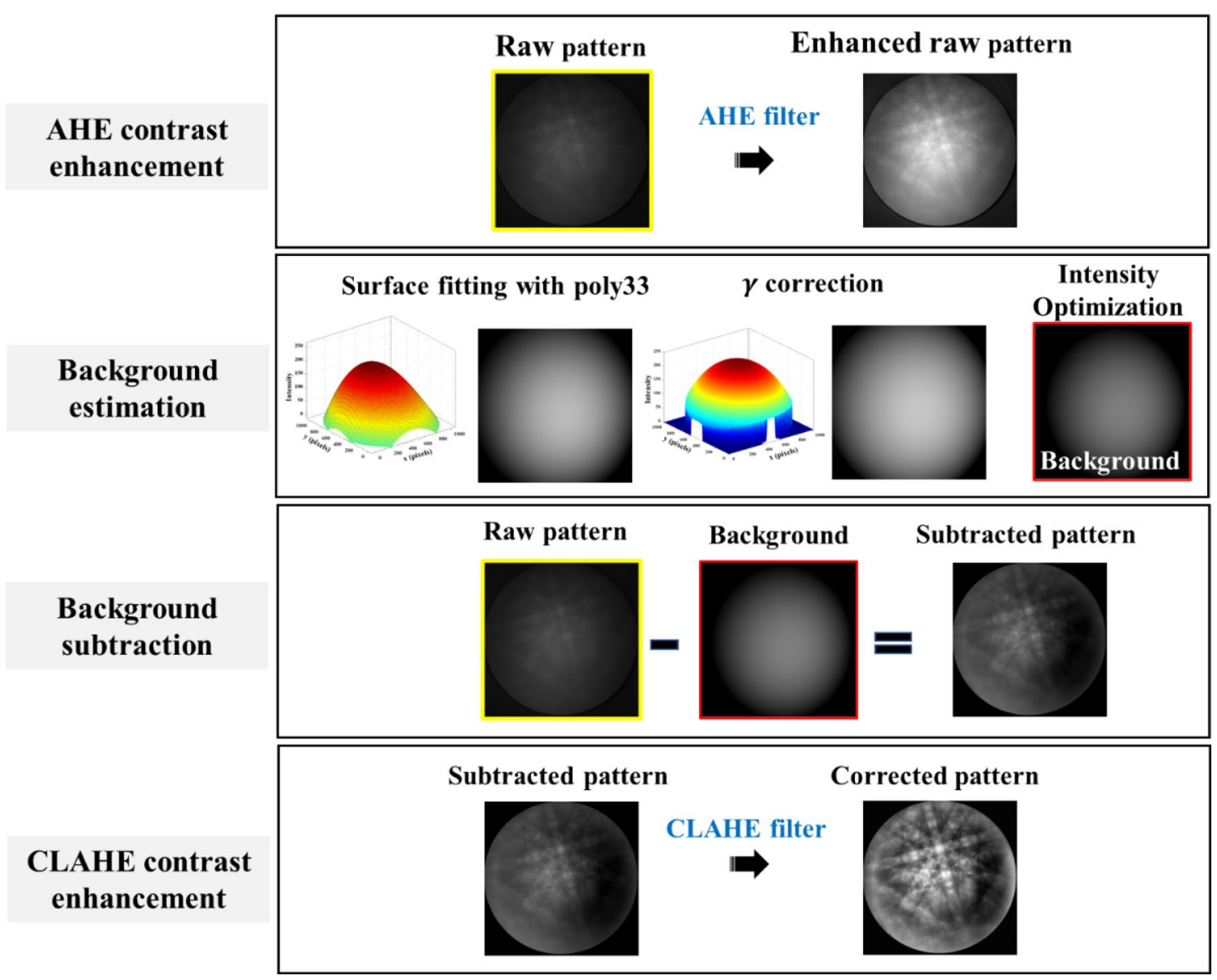

Figure 4. Illustration of the procedure of PF background correction algorithm.

Hence, we use the Gaussian low-pass filter to remove the signal of the low frequency region. The Gaussian lowpass filter is given as follows ${ }^{35}$ :

$$
H(u, v)=e^{\frac{-D^{2}(u, v)}{2 D_{0}^{2}}},
$$

where $D(u, v)$ is the distance from the center of the frequency rectangle, and $D_{0}$ is the cutoff frequency. The generated background uses the FT method, where a cutoff frequency of 10 is selected for the Gaussian low-pass filter.

In the third method, which uses the PF approach, the electron backscatter diffraction patterns are composed of a Kikuchi diffraction pattern (signal) and a smooth background (noise). To obtain a 3D surface, the 2D Kikuchi pattern should be transformed into a 3D Kikuchi curve by converting the $g_{i}$ value of the gray scale at $\left(\mathrm{X}_{\mathrm{i}}, \mathrm{Y}_{\mathrm{i}}\right)$ to the $\mathrm{Z}_{\mathrm{i}}$ value corresponding to a point at $\left(\mathrm{X}_{\mathrm{i}}, \mathrm{Y}_{\mathrm{i}}, \mathrm{Z}_{\mathrm{i}}\right)$ in $3 \mathrm{D}$. The latter can be approximated by a smooth surface with polynomials ${ }^{37}$, which are used for curve fitting techniques and given by the following:

$$
F(x, y)=\sum_{k=0}^{N} \sum_{i=0}^{N-k} p_{i, k} x^{i} y^{k}
$$

where $N$ is the order in the range between 1 and 5 , and $p_{i, k}$ is the coefficient of the polynomial function. The order $\mathrm{N}$ is considered larger than 3 , and the fitting background includes detailed diffraction information, that is, diffraction signals. Thus, the selected value of the order $N$ is 3 , which is called the poly33 model and given as follows:

$$
\text { Poly } 33=F(x, y)=p 00+p 10 * x+p 01 * y+p 20 * x^{2}+p 11 * x * y+p 02 * y^{2}+p 30 * x^{3}+p 21 * x^{2} * y+p 12 * x * y^{2}+p 03 * y^{3} .
$$

The least squares curve fitting technique is used for the solution of the best-fitting curve by minimizing the sum of the least square errors of the data points. Then, the maximum likelihood estimation for the parameter $p_{i j}$ can be obtained by minimizing the chi square. The optimized solution for the $3 \mathrm{D}$ fitting curve follows the least squares error of $\chi^{2}$ given by the following:

$$
\chi^{2}=\sum_{j=1}^{M}\left(z_{j}-\sum_{k=0}^{N} \sum_{i=0}^{N-k} p_{i, k} x^{i} y^{k}\right)^{2},
$$




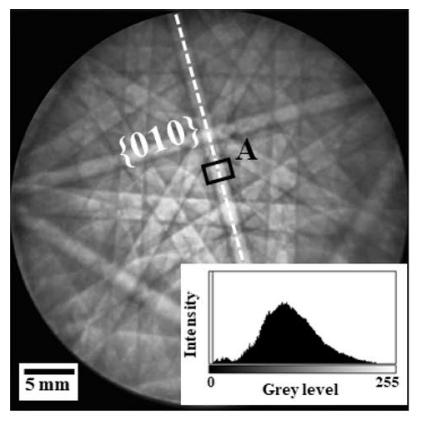

(a)

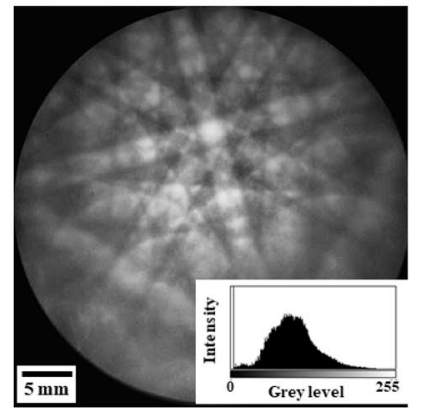

(d)

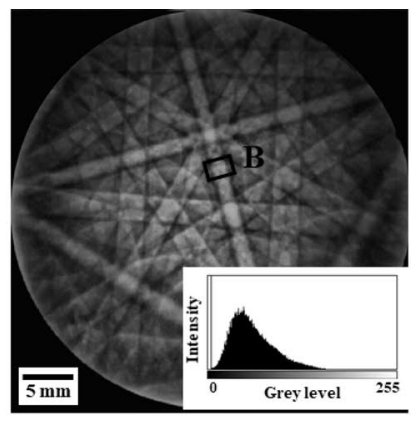

(b)

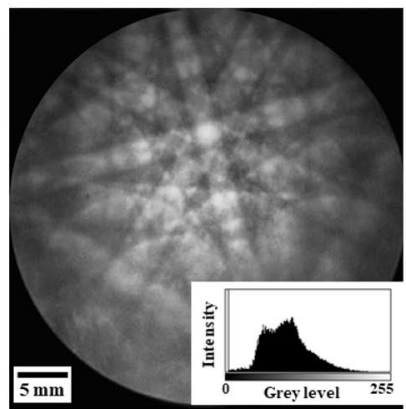

(e)

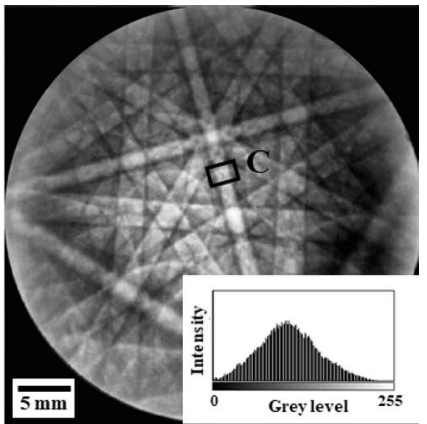

(c)

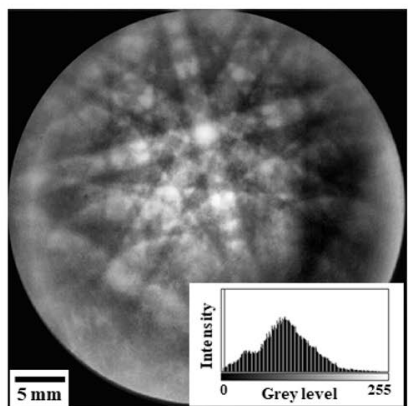

(f)

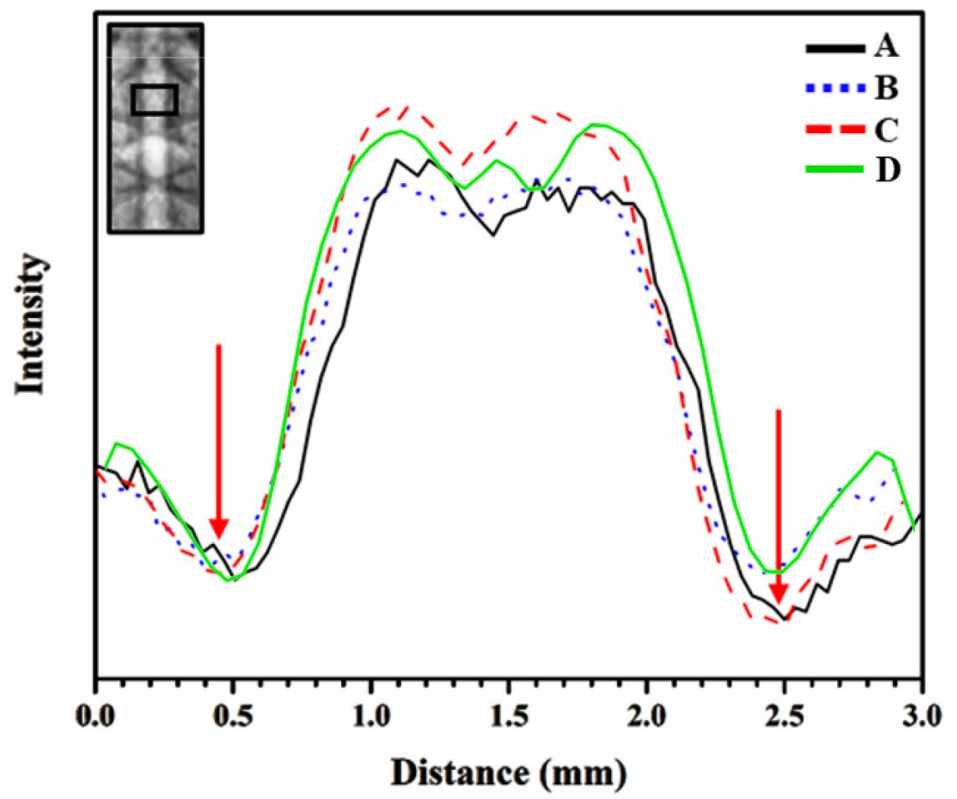

(g)

Figure 5. Kikuchi patterns at 15 and $5 \mathrm{kV}$ after background correction by backgrounds generated by (a,d) PA, (b,e) FT, and (c,f) PF methods. (g) $\{010\}$ Band intensity profiles of Kikuchi patterns at $15 \mathrm{kV}$ after background correction using PA (A), FT (B), and PF (C) methods, where the arrow symbols show the position of the band edges detected using the PF background correction. (Green solid profile D is the simulated band profile of Ni at $15 \mathrm{kV})$. 


\begin{tabular}{|l|l|l|l|l|}
\hline $\mathbf{k V}$ & Index & PA & FT & PF \\
\hline \multirow{3}{*}{$15 \mathrm{kV}$} & PQ & 12,993 & 10,677 & 18,652 \\
\cline { 2 - 5 } & TenV & $5.14 \times 10^{6}$ & $2.92 \times 10^{7}$ & $7.33 \times 10^{7}$ \\
\cline { 2 - 5 } & SSEQ & 35.62 & 35.02 & 34.69 \\
\hline \multirow{3}{*}{$5 \mathrm{kV}$} & PQ & 4921 & 13,699 & 24,062 \\
\cline { 2 - 5 } & TenV & $2.49 \times 10^{6}$ & $4.90 \times 10^{6}$ & $3.41 \times 10^{7}$ \\
\cline { 2 - 5 } & SSEQ & 18.54 & 19.68 & 18.92 \\
\hline
\end{tabular}

Table 2. Indexing values of PQ, TenV, and SSEQ at accelerating voltage of $15 \mathrm{kV}$ and $5 \mathrm{kV}$.

$$
\frac{\partial \chi^{2}}{\partial p_{i, k}}=0
$$

where $M$ is the data number, and $z_{j}$ is the input value at a position of $\left(x_{j}, y_{j}\right)$. The backgrounds were generated by PA, FT, and PF methods, as shown in Fig. 2d,f,h for $15 \mathrm{kV}$ and in Fig. 2e,g,i for $5 \mathrm{kV}$, which are used for the subsequent background correction process.

After the polynomial regression approach, the intensity of the generated background surface can be smaller than that of the raw pattern in $3 \mathrm{D}$. This observation results in a negative value after background correction. To avoid the occurrence of negative values after background correction, a $\gamma$ correction function should be used to compensate for the insufficient intensity of the generated background, and the $\gamma$ correction function is given by the following ${ }^{38}$ :

$$
s=c r^{\gamma}
$$

where $r$ is the gray level of the input, $s$ is that of the output, and $c$ is a constant. The parameters of $\gamma=0.65$ and $c=1$ are used in $\gamma$ correction, where the $\gamma$ value is used to compensate for the insufficient intensity of the raw image.

After $\gamma$ correction and optimization processes, the generated background images in Fig. 3a,c,e reveal an improved contrast when compared with the images without $\gamma$ correction and optimization processes in Fig. 2d,f,h for $15 \mathrm{kV}$. The 3D plots of the background are shown in Fig. 3b,d,f. Thus, the background generation procedures include contrast enhancement, surface fitting, $\gamma$ correction, and background optimization, as shown in Fig. 4 .

Background correction algorithm with PF, PA, and FT methods. After hitting the specimen surface, the primary beam electrons suffer random elastic and inelastic scattering due to energy lose. After a given penetration depth, the electrons scatter at various angles and some electrons diffract following the Bragg's law, resulting in Kikuchi bands. Thus, a relatively low-intensity signal is superimposed with a dominating background signal, as observed on the detector ${ }^{39}$. To solve the problem of low signal-to-noise ratio in EBSD patterns, the background correction algorithm is generally used for the post-processing of EBSD patterns. Thus, we propose a PF background correction algorithm, as shown in Fig. 4: AHE contrast enhancement, background estimation, background subtraction, and contrast-limited adaptive histogram equalization (CLAHE) contrast enhancement.

After background subtraction, CLAHE algorithm is proposed to solve the problem of contrast degradation and to enhance the image contrast in the preprocessing stages. The CLAHE contrast enhancement is applied for PA, FT, and PF methods to compare the image quality after background subtraction, as shown in Fig. 5 . The PF method at $15 \mathrm{kV}$ have the highest value of the PQ and TenV indices at 18,652 and $7.33 \times 10^{7}$, respectively, whereas the SSEQ index has the lowest value of 34.69, as shown in Table 2. These results suggest that the pattern, after the $\mathrm{PF}$ background correction method, reveals clear and sharp images with decreasing noise.

In addition, in the case of $5 \mathrm{kV}, \mathrm{PQ}$ and TenV indices have the highest value of 24,062 and $3.41 \times 10^{7}$, but no significant difference is observed in the SSEQ value for PF method. Here, the PF method provides a bright background image with a broad intensity distribution at $5 \mathrm{kV}$. Therefore, we successfully demonstrate that the PF background correction method in addition to PA and FT methods can effectively extract a background and Kikuchi pattern from raw diffraction patterns.

To evaluate the image quality after background correction, the profiles of $\{010\}$ band width represented by black areas called A, B, and C are shown in Fig. 5a-c for PA, FT, and PF methods, respectively. The simulated edges of the $\{010\}$ Kikuchi band in Fig. $5 \mathrm{~g}$ are compared, and the $\{010\}$ profile of the $\mathrm{C}$ area for the PF method indicates a significant difference between peaks and valleys, that is, a clear band pattern for pattern indexing.

Case study of PF background correction. The spatial resolution of EBSD can be improved at low accelerated voltage for nonconductive materials. However, lowering the beam energy of the electrons reduces the mean free paths of these elastic and inelastic processes rapidly ${ }^{33}$. Moreover, considering nonconductive samples, conductive coating should be required on the sample surface with a thin amorphous layer of gold or platinum to minimize charging effects ${ }^{40}$. Unfortunately, the effects of low accelerated voltage and conductive coating layer decrease the quality of Kikuchi patterns ${ }^{12}$.

We used Ag bicrystals of A and B and coated a Pt layer only on the left side of the white dotted line in Fig. 6g, considering the effects of low accelerated voltage and conductive coating layer. Then, the raw patterns without 


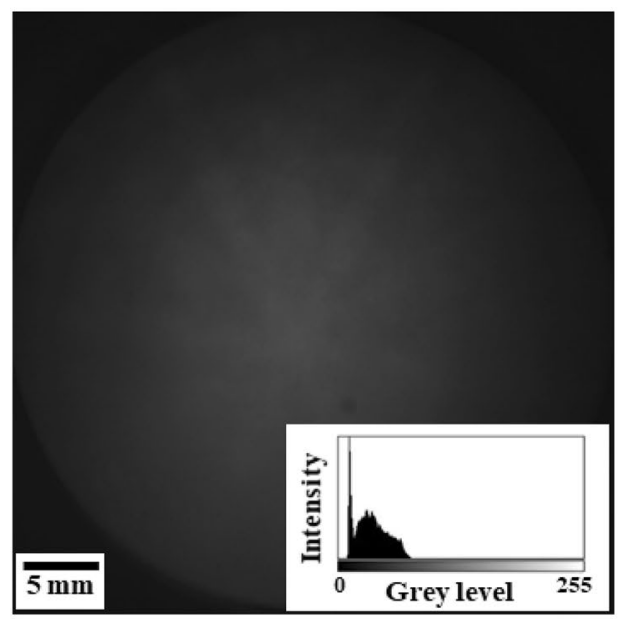

(a)

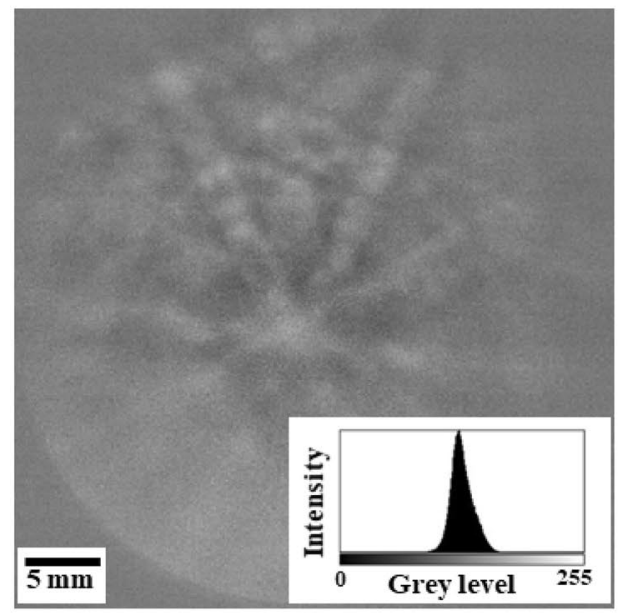

(c)

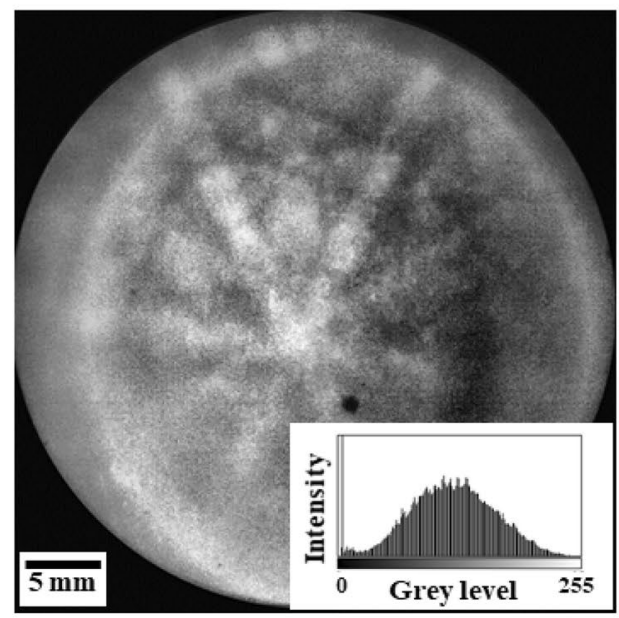

(e)

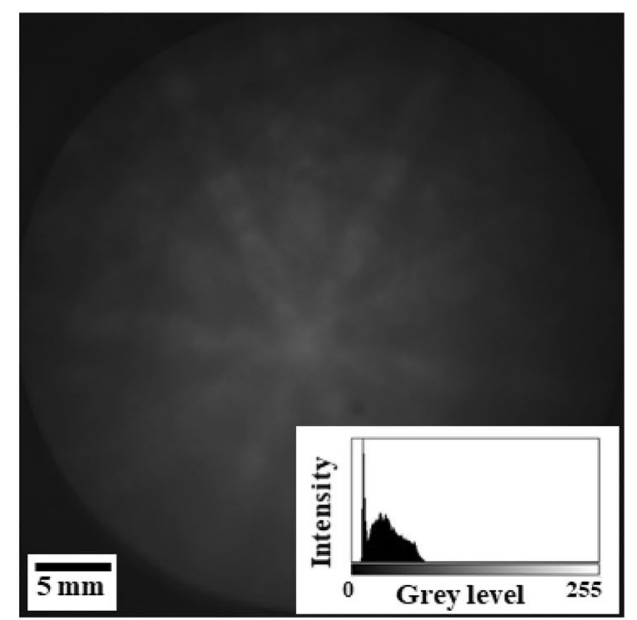

(b)

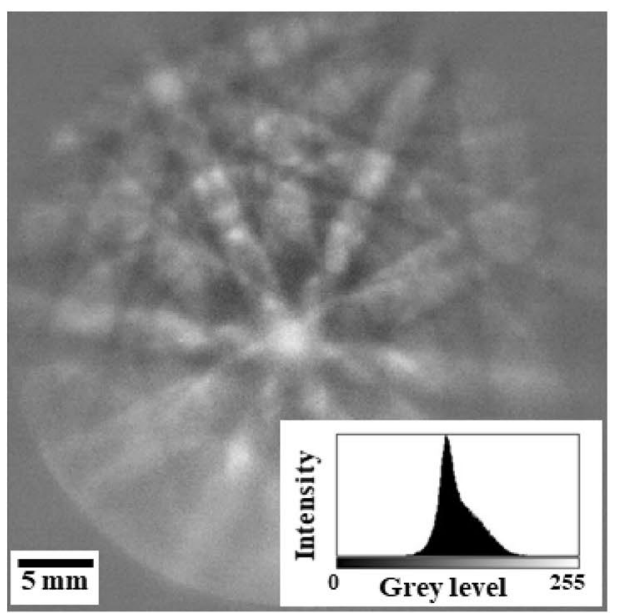

(d)

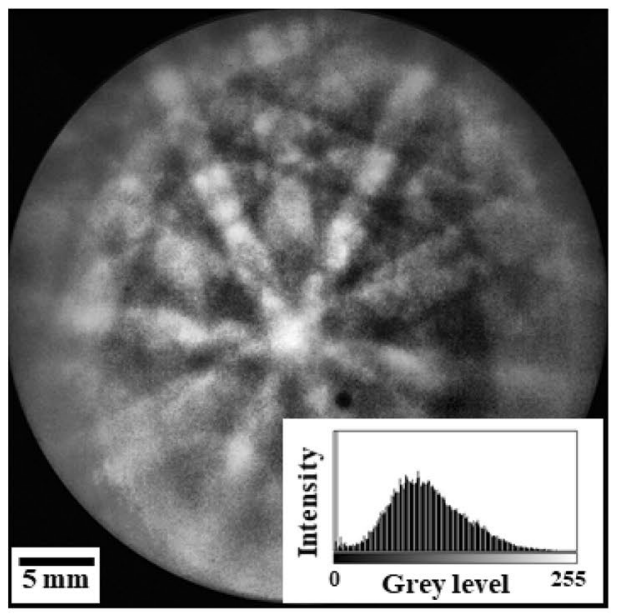

(f)

Figure 6. (a,b) Raw pattern, background-corrected patterns by (c,d) PA and (e,f) PF methods, where (a,c,e) are after $30 \mathrm{~s}$ Pt coating and (b,e,f) are without Pt coating at $5 \mathrm{kV}$. (g) Profiles of the normalized PQ values without and with coating obtained by EBSD line scanning, where the black solid and dash lines are without and with PF background correction, and the white dot line in the lower right image shows the boundary between coated and uncoated zone. 


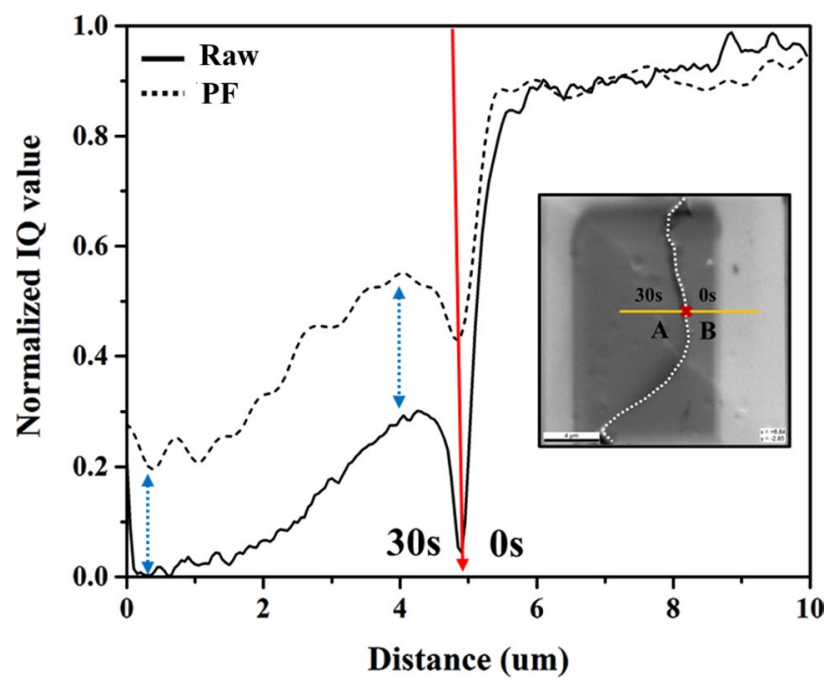

(g)

Figure 6. (continued)

and with coating were used to carry out background correction by PA and PF methods to compare the image quality between both methods. Figure $6 \mathrm{a}, \mathrm{b}$ show the raw patterns with and without $30 \mathrm{~s}$ Pt coating layer on A and B grains. The figures show that the Kikuchi bands became blurred and diffused after Pt coating. Figure $6 \mathrm{c}, \mathrm{d}$ and Fig. 6e,f display the results of Kikuchi patterns with PA and PF background correction method, respectively. The figures show that, for the case without coating, the PQ index does not have significant difference between PA and PF correction; the TenV index is $2.67 \times 10^{6}$ for PA and $2.60 \times 10^{7}$ for PF. In addition, the SSEQ index is 18.63 for PA and 4.95 for PF. After coating, the PQ, TenV, and SSEQ index values are $2319.3,2.49 \times 10^{6}$, and 37.55 for PA and $4789.3,8.07 \times 10^{7}$, and 6.07 for PF. The observations suggest that the contrast and sharpness of $\mathrm{PF}$ were improved compared with those of $\mathrm{PA}$, and $\mathrm{PF}$ decreased noise.

We also used the normalized PQ based on Eq. (3) to quantify the image quality after PF correction and the normalization of PQ by the following ${ }^{41}$ :

$$
P Q_{\text {Normalized }}=\frac{P Q_{\text {value }}-P Q_{\text {min }}}{P Q_{\max }-P Q_{\text {min }}}
$$

where $P Q_{\max }$ and $P Q_{\min }$ are the maximum and the minimum values of $P Q$, respectively. High $P Q$ values indicate good pattern quality and can be better indexed after Hough transform ${ }^{42}$. The profile in Fig. $6 \mathrm{~g}$ shows that the normalized PQ value significantly decreases after coating for both background corrections. Thus, the amorphous coating layer degrades the pattern quality. The difference in the normalized PQ value between PA and $\mathrm{PF}$ corrections reveals no significant change in the case without coating, whereas the normalized PQ value of $\mathrm{PF}$ correction in the case with coating is approximately twice that of PA correction. Hence, the results indicate that the PF background correction can improve the image quality of the coating layer at low accelerated voltage.

\section{Conclusions}

The quality metrics of the diffraction patterns are evaluated using PQ, TenV, and SSEQ indices, which correspond to the clarity, high contrast, and high noise of the patterns, respectively. We propose a PF background correction method to extract background noise and diffraction pattern from raw diffraction patterns under low accelerated voltage or coated sample. After background correction, the PF method offers the best quality, with a PQ value of 24,062 , a TenV value of $3.41 \times 10^{7}$, and an SSEQ value of 18.92 , for diffraction patterns obtained at $5 \mathrm{kV}$ compared with the PA and PF methods. Thus, this proposed PF method can improve the quality of diffraction patterns in EBSD measurements at low accelerating voltages and with coating layer.

\section{Methods}

Acquisition of raw Kikuchi patterns. All the experimental Kikuchi patterns presented in this study were measured with a JEOL 7001 FE-SEM with a field emissions gun and an EDAX/TSL EBSD system equipped with a DigiView CCD camera and a phosphorescent screen with the diameter of $40 \mathrm{~mm}$. The sample was tilted 70 degrees, and EBSD measurements were performed at a working distance of $15 \mathrm{~mm}$. The parameter of Pt coating is $10 \mathrm{~mA}$ using sputter with $30 \mathrm{~s}$. The raw Kikuchi patterns were captured using a $1 \times 1$ binning mode, and the gain value and exposure time were optimized to achieve high-quality patterns. In the line scan of EBSD experiment, the step size is $50 \mathrm{~nm}$. Each raw Kikuchi pattern with a resolution of $1024 \times 1024$ pixels in 8-bit digitization was acquired, and the following image processing procedures were conducted using MATLAB. 
Received: 6 August 2021; Accepted: 13 December 2021

Published online: 10 January 2022

\section{References}

1. Humphreys, F. J. Review Grain and subgrain characterization by electron backscatter diffraction. J. Mater. Sci. 36, 3833-3854. https://doi.org/10.1023/A:1017973432592 (2001).

2. Schwartz, A. J., Kumar, M., Adams, B. L. \& Field, D. P. Electron Backscatter Diffraction in Materials Science (Springer, 2009).

3. Ren, S. X., Kenik, E. A., Alexander, K. B. \& Goyal, A. Exploring spatial resolution in electron back-scattered diffraction experiments via Monte Carlo simulation. Microsc. Microanal. 4, 15-22. https://doi.org/10.1017/S1431927698980011 (1998).

4. Deal, A., Hooghan, T. \& Eades, A. Energy-filtered electron backscatter diffraction. Ultramicroscopy 108, 116-125. https://doi.org/ 10.1016/j.ultramic.2007.03.010 (2007).

5. Pascal, E. et al. Energy-weighted dynamical scattering simulations of electron diffraction modalities in the scanning electron microscope. Ultramicroscopy 187, 98-106. https://doi.org/10.1016/j.ultramic.2018.01.003 (2018).

6. Brodu, E., Bouzy, E. \& Fundenberger, J. J. Diffraction contrast dependence on sample thickness and incident energy in on-axis Transmission Kikuchi Diffraction in SEM. Ultramicroscopy 181, 123-133. https://doi.org/10.1016/j.ultramic.2017.04.017 (2017).

7. Kuo, C. W., Kuo, J. C. \& Wang, S. C. Resolution of transmission electron backscatter diffraction in aluminum and silver: Effect of the atomic number. Ultramicroscopy 193, 126-136. https://doi.org/10.1016/j.ultramic.2018.06.019 (2018).

8. Steinmetz, D. R. \& Zaefferer, S. Towards ultrahigh resolution EBSD by low accelerating voltage. Mater. Sci. Technol. 26, 640-645. https://doi.org/10.1179/026708309X12506933873828 (2013).

9. Chen, D., Kuo, J. C. \& Wu, W. T. Effect of microscopic parameters on EBSD spatial resolution. Ultramicroscopy 111, 1488-1494. https://doi.org/10.1016/j.ultramic.2011.06.007 (2011).

10. Tripathi, A. \& Zaefferer, S. On the resolution of EBSD across atomic density and accelerating voltage with a particular focus on the light metal magnesium. Ultramicroscopy 207, 112828. https://doi.org/10.1016/j.ultramic.2019.112828 (2019).

11. Farrer, J. K., Michae, J. R. \& Carter, C. B. Electron Backscatter Diffraction in Materials Science (Springer, 2000).

12. Wuhrer, R. \& Moran, K. Low voltage imaging and X-ray microanalysis in the SEM challenges and opportunities. IOP Conf. Ser. Mater. Sci. Eng. 109, 012019. https://doi.org/10.1088/1757-899X/109/1/012019 (2016).

13. Liu, J. High-resolution and low voltage FE-SEM imaging and microanalysis in materials characterization. Mater. Charact. 44, 353-363. https://doi.org/10.1016/S1044-5803(99)00076-5 (2000).

14. Callahan, P. G. \& De Graef, M. Dynamical electron backscatter diffraction patterns Part I: Pattern simulations. Microsc. Microanal. 19, 1255-1265. https://doi.org/10.1017/S1431927613001840 (2013).

15. Winkelmann, A. Dynamical effects of anisotropic inelastic scattering in electron backscatter diffraction. Ultramicroscopy 108, 1546-1550. https://doi.org/10.1016/j.ultramic.2008.05.002 (2008).

16. Field, D. P. Improving the spatial resolution of EBSD. Microsc. Microanal. https://doi.org/10.1017/s1431927605506445 (2005).

17. Winkelmann, A. \& Vos, M. The role of localized recoil in the formation of Kikuchi patterns. Ultramicroscopy 125, 66-71. https:// doi.org/10.1016/j.ultramic.2012.11.001 (2013).

18. Omoto, K., Tsuda, K. \& Tanaka, M. Simulations of Kikuchi patterns due to thermal diffuse scattering on MgO crystals. J. Electron Microsc. 51, 67-78. https://doi.org/10.1093/jmicro/51.1.67 (2002).

19. Wang, Z. L. Thermal diffuse scattering in sub-angstrom quantitative electron microscopy-phenomenon, effects and approaches. Micron 34, 141-155. https://doi.org/10.1016/S0968-4328(03)00024-6 (2003).

20. Winkelmann, A. et al. Diffraction effects and inelastic electron transport in angle-resolved microscopic imaging applications. J. Microsc. 267, 330-346. https://doi.org/10.1111/jmi.12571 (2017).

21. Deal, A., Tao, X. \& Eades, A. EBSD geometry in the SEM: Simulation and representation. Surf. Interface Anal. 37, 1017-1020. https://doi.org/10.1002/sia.2115 (2005)

22. Wright, S. I., Nowell, M. M., de Kloe, R., Camus, P. \& Rampton, T. Electron imaging with an EBSD detector. Ultramicroscopy 148, 132-145. https://doi.org/10.1016/j.ultramic.2014.10.002 (2015).

23. Schwarzer, R. A. \& Sukkau, J. Electron back scattered diffraction- current state, prospects and comparison with X-ray diffraction texture measurement. Banaras Metall. 18, 1-11 (2013).

24. Payton, E. J. \& Nolze, G. The backscatter electron signal as an additional tool for phase segmentation in electron backscatter diffraction. Microsc. Microanal. 19, 929-941. https://doi.org/10.1017/S1431927613000305 (2013).

25. Tao, X. \& Eades, A. Errors, artifacts, and improvements in EBSD processing and mapping. Microsc. Microanal. 11, 79-87. https:// doi.org/10.1017/S1431927605050099 (2005).

26. Pizer, S. M. E. A. Adaptive histogram equalization and its variations. Comput. Vis. Graph. Image Process. 39, 355-368. https://doi. org/10.1016/S0734-189X(87)80186-X (1987).

27. Britton, T. B., Tong, V. S., Hickey, J., Foden, A. \& Wilkinson, A. J. AstroEBSD: Exploring new space in pattern indexing with methods launched from an astronomical approach. J. Appl. Crystallogr. 51, 1525-1534. https://doi.org/10.1107/s16005767180103 73 (2018).

28. Wilkinson, A. J., Meaden, G. \& Dingley, D. J. High-resolution elastic strain measurement from electron backscatter diffraction patterns: New levels of sensitivity. Ultramicroscopy 106, 307-313. https://doi.org/10.1016/j.ultramic.2005.10.001 (2006).

29. Wright, S. I. \& Nowell, M. M. EBSD image quality mapping. Microsc. Microanal. 12, 72-84. https://doi.org/10.1017/S143192760 6060090 (2006).

30. Kunze, K., Wright, S. I., Adams, B. L. \& Dingley, D. J. Advances in automatic EBSP single orientation measurements. Textur. Microstruct. 20, 41-54. https://doi.org/10.1155/TSM.20.41 (1993).

31. Pech-Pacheco, J. L., Cristobal, G., Chamorro-Martinez, J., Fernandez-Valdivia, J. Diatom autofocusing in brightfield microscopy: a comparative study. Proceedings 15th International Conference on Pattern Recognition 3, 314-317, doi:https://doi.org/10.1109/ ICPR.2000.903548 (2000).

32. Liu, L., Liu, B., Huang, H. \& Bovik, A. C. No-reference image quality assessment based on spatial and spectral entropies. Sign. Process. Image Commun. 29, 856-863. https://doi.org/10.1016/j.image.2014.06.006 (2014).

33. Gonzalez, R. C. Digital Image Processing 3rd edn. (Pearson, 2008).

34. Shapiro, L. G. \& Stockman, G. C. Computer Vision (Prentice Hall, Berlin, 2001).

35. Gonzalez, R. C. \& Woods, R. E. Digital Image Processing 187-213 (Addison Wesley, 1992).

36. Gallagher, T. A., Nemeth, A. J. \& Hacein-Bey, L. An introduction to the Fourier transform: Relationship to MRI. AJR Am. J. Roentgenol. 190, 1396-1405. https://doi.org/10.2214/AJR.07.2874 (2008).

37. Balch, S. J. \& Thompson, G. T. An efficient algorithm for polynomial surface fitting. Comput. Geosci. 15, 107-119. https://doi.org/ 10.1016/0098-3004(89)90058-7 (1989).

38. Bhandari, A. K., Kumar, A. \& Singh, G. K. Improved knee transfer function and gamma correction based method for contrast and brightness enhancement of satellite image. AEU-Int. J. Electron. C. 69, 579-589. https://doi.org/10.1016/j.aeue.2014.11.012 (2015).

39. Winkelmann, A. Principles of depth-resolved Kikuchi pattern simulation for electron backscatter diffraction. J. Microsc. 239, 32-45. https://doi.org/10.1111/j.1365-2818.2009.03353.x (2010).

40. Saowadee, N., Agersted, K. \& Bowen, J. R. Effects of focused ion beam milling on electron backscatter diffraction patterns in strontium titanate and stabilized zirconia. J. Microsc. 246, 279-286. https://doi.org/10.1111/j.1365-2818.2012.03616.x (2012). 
41. Wu, J., Garcia, C. I., Hua, M. \& Deardo, A. J. Image quality analysis: A new method of characterizing microstructures. ISIJ Int. 45, 254-262. https://doi.org/10.2355/isijinternational.45.254 (2005).

42. Wright, S. I. \& Adams, B. L. Automatic analysis of electron backscatter diffraction patterns. Metall. and Mater. Trans. A. 23, 759-767. https://doi.org/10.1007/BF02675553 (1992).

\section{Acknowledgements}

The authors would gratefully like to thank the Ministry of Science and Technology for supporting the fund of the project under MOST 107-2221-E-006-018 and MOST 109-2221-E-006-131-MY2.

\section{Author contributions}

Y.-Y.T.: conceptualization, methodology, software, formal analysis, investigation, writing-original draft, visualization. Y.-C.P.: methodology, software, formal analysis, writing — original draft, visualization. J.-C.K.: conceptualization, methodology, validation, resources, data curation, writing—original draft, writing—review \& editing, supervision. All authors reviewed the manuscript.

\section{Competing interests}

The authors declare no competing interests.

\section{Additional information}

Correspondence and requests for materials should be addressed to J.-C.K.

Reprints and permissions information is available at www.nature.com/reprints.

Publisher's note Springer Nature remains neutral with regard to jurisdictional claims in published maps and institutional affiliations.

(c) (i) Open Access This article is licensed under a Creative Commons Attribution 4.0 International License, which permits use, sharing, adaptation, distribution and reproduction in any medium or format, as long as you give appropriate credit to the original author(s) and the source, provide a link to the Creative Commons licence, and indicate if changes were made. The images or other third party material in this article are included in the article's Creative Commons licence, unless indicated otherwise in a credit line to the material. If material is not included in the article's Creative Commons licence and your intended use is not permitted by statutory regulation or exceeds the permitted use, you will need to obtain permission directly from the copyright holder. To view a copy of this licence, visit http://creativecommons.org/licenses/by/4.0/.

(C) The Author(s) 2022 